\title{
PROBLEMAS DO COTIDIANO QUE ENSINAM GEOMETRIA
}

Christian Luz Pelissari de Oliveira, Richard Mariano de Souza Silva, Fernando Pereira de Souza, Eugenia Brunilda Opazo Uribe

Universidade Federal do Mato Grosso do Sul - UFMS, Curso de Licenciatura em Matemática, Três Lagoas, MS. E-mail: richard.mariano.mat@gmail.com.

Agência de Fomento: Programa de Educação Tutorial (PET)

\section{RESUMO}

O objetivo do presente trabalho é apresentar um grupo de problemas do cotidiano que podem ser utilizados em sala de aula para o ensino de diversos tópicos de Geometria. Os problemas escolhidos foram publicados pela revista do Professor de Matemática em edições distintas e foram estudados como parte das atividades de um projeto de pesquisa coletivo, desenvolvido no período 2015-2016, no âmbito do Programa de Educação Tutorial, denominado "Matemática, Aplicações e Interdisciplinaridade". Explorar e resolver estes problemas permitiu uma discussão sobre os conteúdos envolvidos, estratégias de resolução, possíveis variações dos problemas originais, bem como um trabalho integrado entre PETianos das diversas séries do Curso de Licenciatura em Matemática.

Palavras-Chave: Ensino de Matemática, Geometria, Resolução de Problemas, Problemas do cotidiano, Aplicações da Matemática.

\section{PROBLEMS OF DAILY LIFE THAT TEACH GEOMETRY}

\begin{abstract}
The objective of this work is to presente a group of everyday problems that can be used in the classroom for teaching geometry topics. The problems were published by journal of mathematics teacher in separate editions and were studied as part of a collective research Project, developed in the period 2015-2016, $\mathrm{n}$ in the Tutorial Educational Program, called "math, and Interdisciplinary Applications". Explore and solve these problems allowed a discussion of the content involved, resolution strategies, possible variations of the original problems, as well as an integrated work between PETianos of the various grades of course degree in mathematics.
\end{abstract}

Keywords: Teaching of Mathematics, Geometry, Problem Resolution, Problems of Daily Life, Applications of Mathematics. 


\section{INTRODUÇÃO}

O trabalho apresenta resultados e um dos tópicos estudados no projeto de pesquisa coletivo "Matemática, Aplicações e Interdisciplinaridade", desenvolvido pelo Grupo PET Conexões de Saberes - Matemática do Campus de Três Lagoas da Universidade Federal de Mato Grosso do Sul no período 2015 - 2016. O tópico foi desenvolvido com o intuito de explorar diversos problemas do cotidiano que permitem abordar conteúdos de Geometria. Nesse trabalho serão abordados três problemas, que, para a sua resolução utilizam conhecimentos de Geometria Plana, Geometria Espacial e Trigonometria.

\section{METODOLOGIA}

O trabalho foi desenvolvido através de um estudo teórico sobre Geometria e sobre Resolução de Problemas, que incluiu apresentação de seminários semanais e resolução de exercícios. Foi utilizado também o programa gráfico SKETCHUP, programa de maquete eletrônica, para fazer todas as figuras utilizadas, de maneira a entender melhor cada uma das etapas da resolução dos problemas estudados.

\section{RESULTADOS}

O primeiro problema aborda o cálculo da área do telhado de um depósito de forma retangular e a cobertura em forma de arco de circunferência. $O$ segundo problema aborda 0 trabalho de um construtor que precisa construir uma janela ornamental aproveitando um pedaço de vidro retangular que apresenta 10 bolhas de ar como defeito, sabendo que não há três bolhas alinhadas entre si, nem duas delas com algum vértice do retângulo, ou umas delas com dois vértices do retângulo. $O$ terceiro problema discute como estabelecer com precisão a forma e a capacidade de um armazém utilizado para estocar milho a granel. $O$ armazém em questão tem forma poliédrica, assim, através de cortes horizontais e transversais, podemos destacar algumas figuras geométricas conhecidas e a partir delas fazer o cálculo do volume e finalmente a capacidade de estocagem procurada.

\section{Cálculo da área do telhado de um depósito}

O primeiro problema relata que um construtor pretende construir um grande depósito, de forma retangular, conforme as dimensões da figura abaixo:

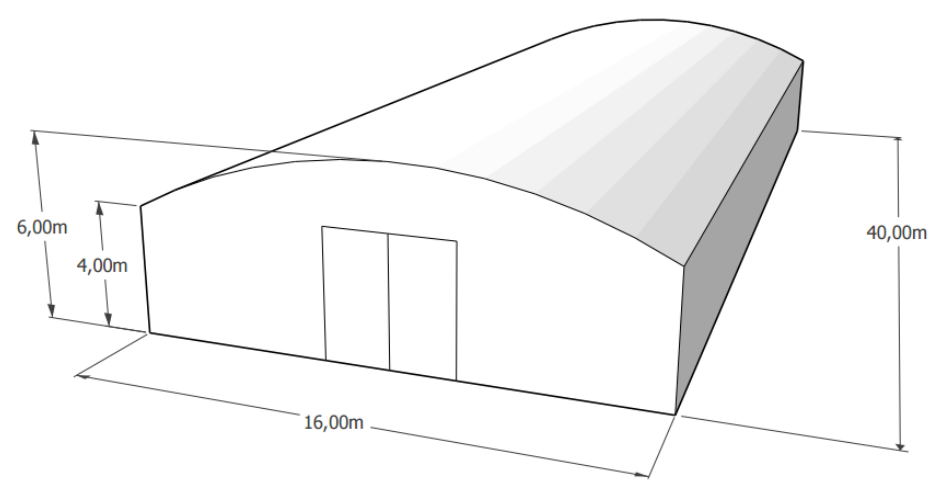

A cobertura do depósito terá um formato de arco de circunferência, com $6 \mathrm{~m}$ de altura máxima, no centro do vão. O construtor gostaria de poder calcular a área do telhado para ter uma base de quanto irá gastar.

A largura do telhado é de $16 \mathrm{~m}$, e a altura dele em relação as paredes do depósito é de $2 \mathrm{~m}$, a figura abaixo foi obtida a partir da figura do depósito: 


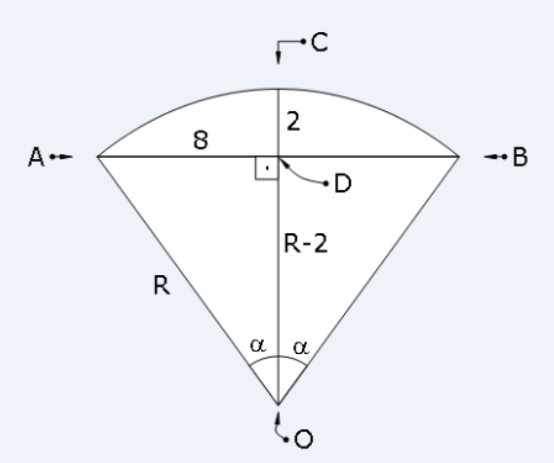

Temos um arco de circunferência $\overline{A C B}$ e dois triângulos retângulos $\triangle A D O \cong B D O$. Assim, aplicando o teorema de Pitágoras ao triângulo AOD temos,

$$
R^{2}=8^{2}+(R-2)^{2} \text { ou } 4 R=68,
$$

e portanto teremos $R=17 \mathrm{~m}$.

No mesmo triângulo retângulo, temos que:

$$
\operatorname{sen} \propto=\frac{A D}{A O}=\frac{8}{17} \cong 0,470
$$

Logo, $\operatorname{arc}(\operatorname{sen}(\propto))=\propto \cong 28^{\circ}$ e, portanto $2 \propto \cong A \hat{O} B \cong 56^{\circ}$.

Além disso, temos que numa circunferência, as medidas dos arcos são diretamente proporcionais às medidas dos ângulos correspondentes, segue que:

$$
\frac{\widehat{A C B}}{56}=\frac{2 \pi R}{360} \quad \text { ou } \quad \widehat{A C B}=\frac{56 \cdot 2 \pi \cdot 17}{360} \cong 16,6 m
$$

Portanto $\overline{A C B} \cong 16,6 m$.

Como a superfície do telhado é parte de uma superfície cilíndrica, planificando-a obtemos um retângulo de lados $16,6 \mathrm{~m}$ e $40 \mathrm{~m}$.

Portanto, encontramos finalmente, que a área do telhado procurada é $A_{\text {telhado }} \cong$

$$
16,6 \cdot 40=664 m^{2} \text {. }
$$

\section{Construção de uma janela ornamental}

Para o segundo problema, imaginaremos que para construir uma janela ornamental, um operário precisa de pedaços triangulares de vidro. Ele pretende aproveitar um vidro retangular defeituoso, com 10 bolhas de ar, sendo que não há 3 bolhas alinhadas em si, nem duas delas com algum vértice do retângulo, ou uma delas com 2 vértices do retângulo. Para evitar bolhas de ar em seu projeto final, ele decidiu cortar os pedaços triangulares com os vértices coincidindo ou com uma bolha de ar, ou com um dos cantos de vidro original, quantos pedaços triangulares ele teve que cortar?

Observemos que, se tivermos uma bolha, serão formados 4 triângulos, se tivermos duas bolhas serão formados 6 triângulos, para 3 bolhas teremos 8 triângulos e assim por diante.
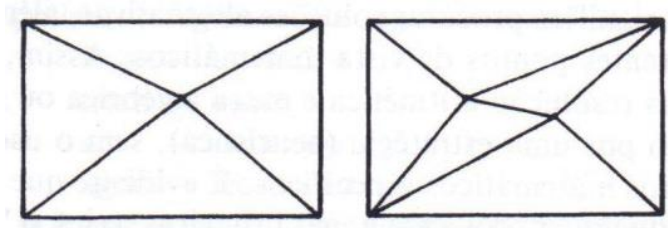

Levando em conta que a soma dos ângulos internos de um triangulo é $180^{\circ}$, a soma $\mathrm{S}$ das medidas dos ângulos internos de todos os triângulos formados será $180^{\circ}$ vezes o número de triângulos que teremos.

Por outro lado, teremos que $S$ dado pela soma dos ângulos internos em volta de cada bolha mais a soma dos 4 ângulos retos dos vértices do vidro, será

$$
\mathrm{S}=360^{\circ} \cdot 10+90^{\circ} \cdot 4=3960^{\circ} \text {, }
$$

Fazendo $3960^{\circ} / 180^{\circ}$, obtemos que, o número de triângulos será 22 . 


\section{Cálculo da capacidade de um armazém}

Já nosso terceiro problema trata de um armazém denominado graneleiro construído há 30 anos, embora de sólida e perfeita construção, o mesmo carecia de especificações precisas sobre sua forma e capacidade, pois na entressafra, quando o milho é vendido e retirado do graneleiro, a "sobra" é rateada entre os cooperados, surgindo então a necessidade de estabelecer, com precisão, a forma e a capacidade do graneleiro.

A solução consiste em dividir o armazém em partes, de modo a poder analisar a capacidade do mesmo através de volumes de sólidos conhecidos.

Neste problema nosso armazém tem forma poliédrica, com as dimensões indicadas na figura abaixo,
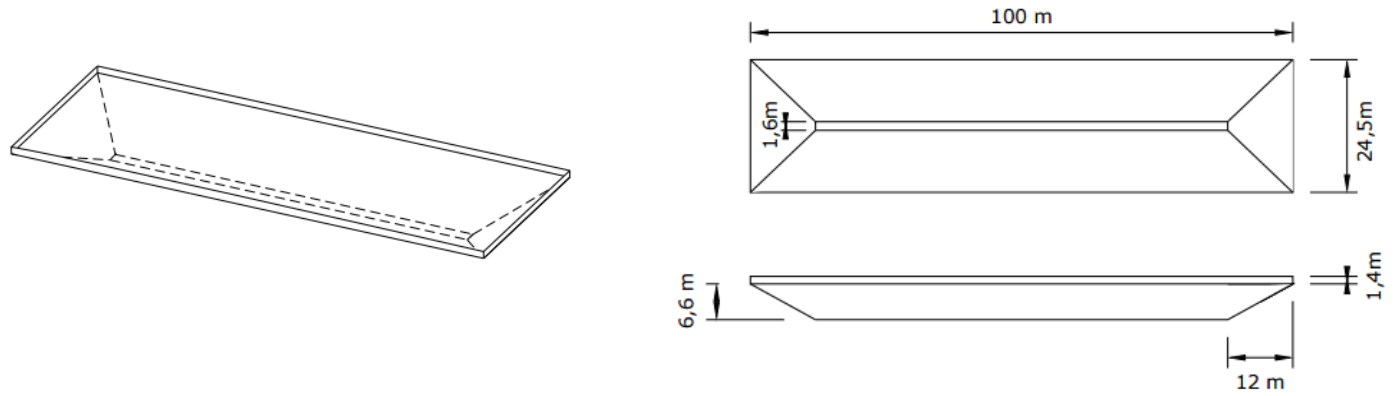

Iniciamos fazendo um corte horizontal, de maneira a destacarmos do sólido um paralelepípedo retângulo como o da figura abaixo,

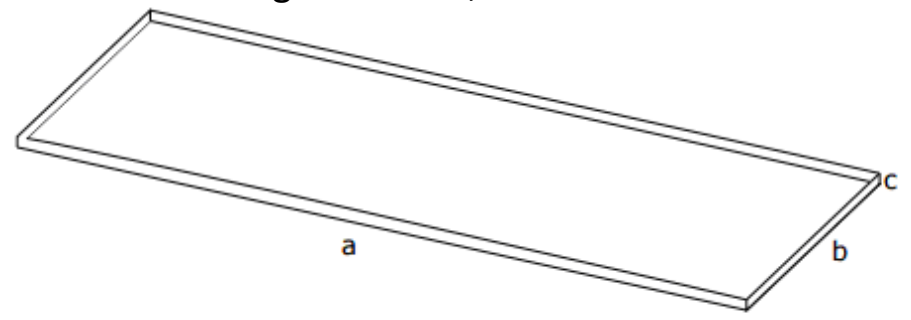

Para o qual teremos as medidas, $a=100 m, b=24,5 m$ e $c=1,4 m$. Lembrando que, o volume será dado por $V=a \cdot b \cdot c$, teremos

$$
V=100 \cdot 24,5 \cdot 1,4=3430 \mathrm{~m}^{3} .
$$

$\mathrm{Na}$ parte restante do armazém, fazemos dois cortes transversais, obtendo três partes, nas extremidades duas partes simétricas e no centro um prisma de base trapezoidal como o da figura abaixo:

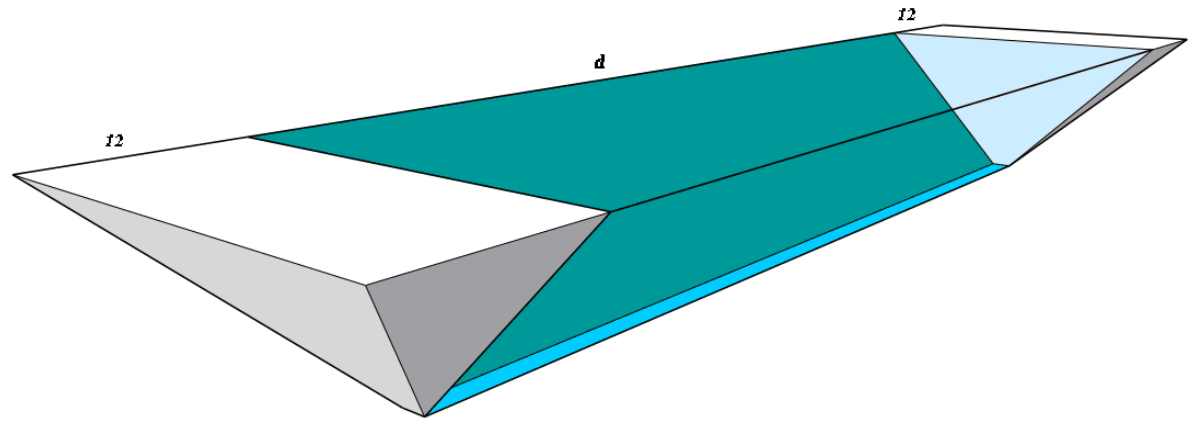

e, cujas medidas estão representadas na seguinte figura, 


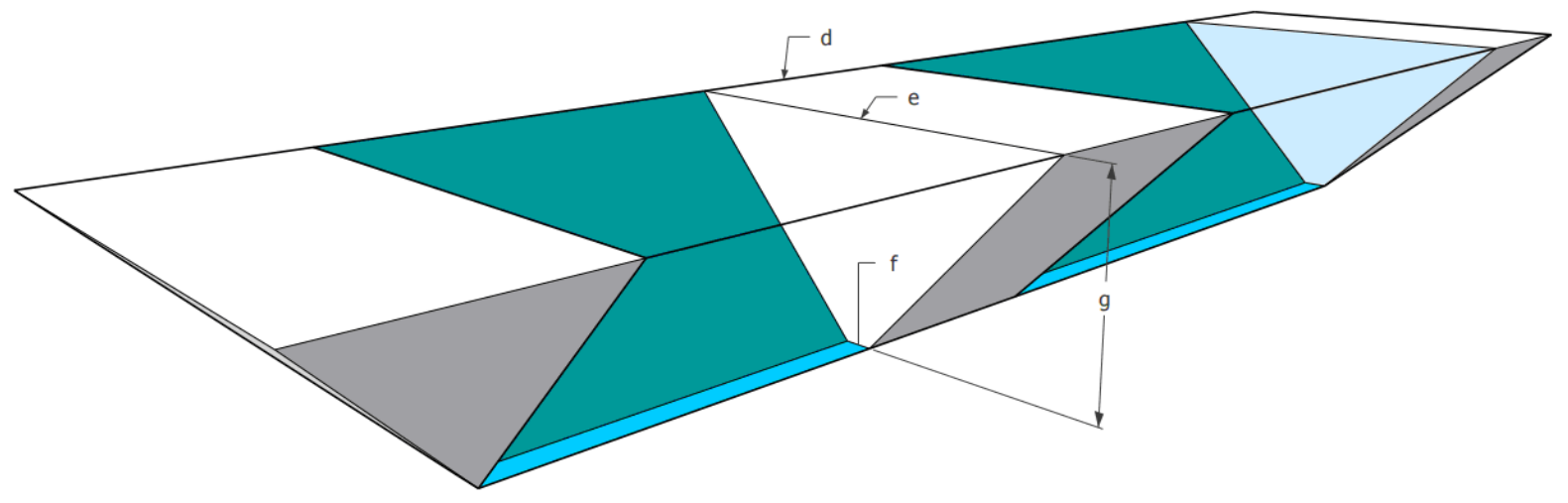

onde, $d=100 m-2 \cdot 12 m=76 m, e=24,5 m, f=1,6 m, g=6,6 m$. Como o volume do prisma trapezoidal será $\quad V_{2}=\frac{f+e}{2} \times g \times d=\frac{1,6+24,5}{2} \times 6,6 \times 76=6545,88$, obtendo assim, $V_{2}=6545,88 m^{3}$.

Como as pontas que restam são simétricas, podem ser decompostas em um prisma de base triangular e duas pirâmides (simétricas) de base retangular:

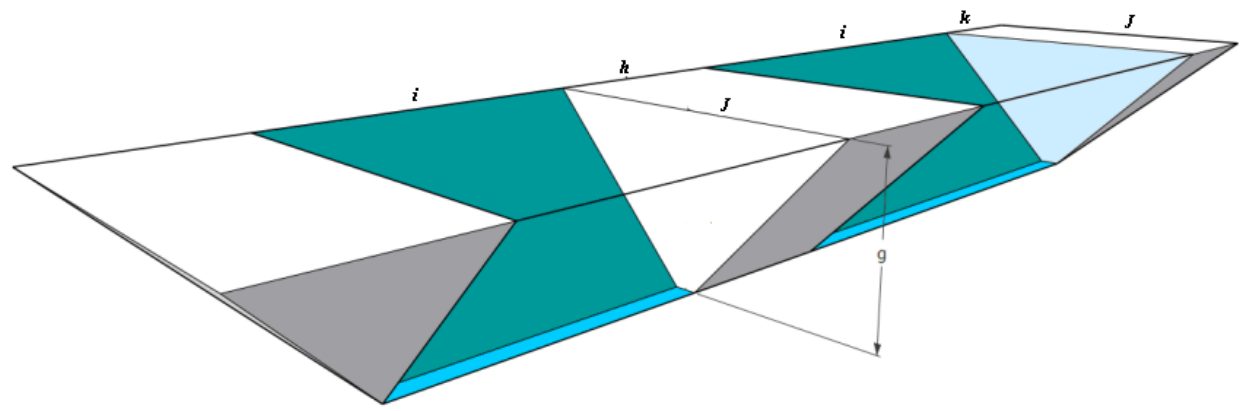

e considerando que, $h=1,6 m, i=g=6,6 m, j=12 m$, e que $k$ pode ser calculado como $k=\frac{24,5-1,6}{2}=11,45$.

O Volume do prisma triangular é dado pela fórmula $V_{3}=\frac{i \cdot j}{2} \cdot h$, onde i representa a altura do triângulo da base do prisma, j representa o tamanho do lado do triângulo de onde foi prolongada a altura e $\mathrm{h}$ representa a altura do prisma. Teremos então que $V_{3}=63,36 \mathrm{~m}^{3}$.

O volume da pirâmide triangular é dado pela fórmula $V_{4}=\frac{j \cdot k \cdot i}{3}$, então $V_{4}=\frac{12 \times 11,45 \times 6,6}{3}=302,28 \mathrm{~m}^{3}$.

Desta forma, podemos obter o volume total do armazém pela soma dos volumes de cada umas das partes

Obtendo como resultado

$$
V_{G}=V_{1}+V_{2}+2\left(V_{3}+2 V_{4}\right)
$$

$$
V_{G}=3430+6545,88+2(63,36+2 \cdot 302,28)=11.311,72 m^{3} .
$$

Portanto, o volume do milho que o armazém comporta quando raso é de $11311,72 \mathrm{~m}^{3}$, Considerando que, os manuais teóricos indicam que o peso especifico do milho é de $0,750 t / \mathrm{m}^{3}$, portando a capacidade total do armazém quando raso será de

$$
C_{R}=11.311,72 \times 0,750 \cong 8483,79 t
$$




\section{DISCUSSÃO}

A utilização de problemas para o desenvolvimento de conteúdos é uma ferramenta muito poderosa e utilizada tanto no ensino básico como no ensino superior. No caso específico desse trabalho, os problemas permitiram fazer revisão de conceitos básicos de Geometria, incluindo cálculo de volumes e áreas, além de uma revisão de sólidos geométricos e suas principais características.

\section{CONCLUSÕES}

Consideramos nosso objetivo atingido já que foi possível explorar diversos conceitos, tanto de Geometria Plana como Espacial, através da utilização de problemas do cotidiano. Além disso, os alunos participantes do projeto poderão utilizar os conteúdos desenvolvidos na sua futura vida profissional, na elaboração de projetos multidisciplinares e inclusive em atividades extraclasse, buscando a aproximação dos alunos com a disciplina de Matemática.

\section{REFERÊNCIAS}

Freiria, A. A. A capacidade do graneleiro. Revista do Professor de Matemática No. 15. Sociedade Brasileira de Matemática. 1989.

IMENES, Luiz Marcio.. Revista do Professor de Matemática - número 13. SBM. 1988

Nasser, L. Um problema: resolução e exploração. Revista do Professor de Matemática No. 15. Sociedade Brasileira de Matemática. 1989. 